\title{
The Fluid Concept of Community Well-Being
}

\author{
Karim W.F. Youssef*
}

Book Review: Kee, Youngwha, Lee, Seung Jong, \& Phillips, Rhonda (Eds.). (2016). Social Factors and Community Well-Being. Switzerland: Springer International Publishing.

The book is an edited volume that comprises six chapters that explore the construct of community well-being (CWB). Chapter contributions are from the $3^{\text {rd }}$ International Forum on Community Well-Being that took place in Seoul, South Korea from June $23^{\text {rd }}$ to $25^{\text {th }}$, 2014. This book fulfills the purpose of spurring the need for more research on the subject of social factors and their impact on community well-being. The first two chapters take a theoretical approach in exploring the construct of community well-being, while the remaining four chapters take a practical approach and focus on empirical case studies.

HeeKyung Sung and Rhonda Phillips in Chapter One aim to capture the concept of community well-being in a comprehensive and integrative way. To do that, Sung and Phillips look at four dimensions: the human, the social, the economic, and the environmental, dimensions that are roughly paralleled to four theories and to four related concepts of community well-being. The four theories are: systems theory, bottom-up spillover theory, needs theory, and social capital theory. The four concepts are: physical well-being, happiness/life satisfaction, quality of life, and community development. Sung and Phillips synthesize the overall framework of the four dimensions, four theories, and four related concepts using a hierarchical pyramid of concepts involving the human, economic, social, and environmental dimensions. Given the diversity of these related concepts and dimensions, the framework, though simple in its adoption of the basic form of a pyramid, reads nevertheless as ambitious in representing the explanatory power of the framework and the potential network of relations that would tie the concepts together. Sung and Phillips adopt the form of the pyramid as a simplistic and ambitious way to straddle inductive and deductive approaches that they understand as two complementary approaches inherent in the construct of CWB. The explanatory power of the pyramid form, divided in horizontal and hierarchical layers, resides in representing the dynamics of a systems approach. For instance, the human dimension is cast with an inductive approach that emphasizes bottom-up processes of community development. The economic dimension is cast within social capital theory. The social dimension is understood as satisfying needs in various domains (family, leisure, work, etc.). The environmental dimension is cast within a systems paradigm where there is a difference between 'internal' and 'external' relations of community. As appealing as the systems paradigm may be to the authors in pinning down the fluid construct of CWB, one wonders if another form or dynamic would have offered a more vivid framework for the relational complexity embedded within the construct of community well-being. At the end of the chapter, the reader is left wanting when contemplating the pyramid form in tying aspects, dimensions and values of CWB in a tangible, rather than a fluid, way. For instance, the 'human' in the pyramid form is represented both as one of the four facets of the quadrilateral pyramid, as well as one of the domains in the hierarchical horizontal divisions of the pyramid. The juxtaposition between the facets and the layers needed more explanation.

Seung Jong Lee and Yunji Kim in Chapter Two introduce inter-subjective CWB and compare it to quality of life construct (QOL) on the basis that the former involves objective indicators at the community level, while the latter involves subjective indicators at the individual level. There seems to be some vagueness in trying to differentiate between objective and inter-subjective CWB. The basis of the authors' argument is that the inter-subjective lends itself to being objective (i.e., collective) more than mere individual indicators measuring individual well-being (IWB). The authors support this assumption by arguing that the ability to trade places with the other, in the inter-subjective approach, gives more objectivity when evaluating satisfaction from available resources than relying merely on indicators at the individual level. However, the case for supporting inter-subjective CWB over subjective CWB was not fully supported in the chapter, as it was only proved by means of a test of correlation for questionnaire items (p.33).

Youngwha Kee and Chaebong Nam in Chapter Three ask if there is an ontological relation between sense of community (SOC) and CWB. The chapter compares two disparate communities, that of Sungmisan with a population of 2,000 people versus Mapo, having a population of 400,000 . Why the authors compared these two communities despite the wide disparity in population between them remains a methodological question. A survey questionnaire was conducted and comprised 34 indicators for individual well-being (IWB), two questions for sense of community, and additional questions with the purpose of evaluating local living conditions and subjective CWB. The level of subjective CWB was impacted by objective factors of CWB and by subjective factors of satisfaction with living in the community. Sungmisan was found to have a higher individual well-being (IWB) and higher sense of community, while Mapo was found to have a higher 
subjective CWB. These results should be surprising to urban planners and policy makers as smaller sized communities, though evidenced in the literature to have a higher sense of community than larger communities, did not have a higher sense of collective well-being. Astonishingly put, a higher sense of community did not translate into a higher sense of community well-being when comparing a small community to a larger one. This calls for more research into the reasons behind the higher sense of CWB for larger communities over smaller ones. The intent of including this chapter in the book seems to be to disrupt the inclusion of the concept of sense of community within the pyramid of concepts related to CWB as presented in Chapter One. After reading the chapter, one wonders, however, why the authors did not operationalize and establish a measurement scale for sense of community, especially given that sense of community is the main focus of the chapter.

Geoffrey Woolcock in Chapter Four addresses children's well-being at the scale of a community or neighbourhood. Woolcock emphasizes the use of data as a means to direct community engagement with issues that impact the community especially with regard to data on children. The city of Bendigo, Australia is used as a case study for explaining the process that led to establishing a set of indicators for children, families, and communities. Indicators for strategic planning were filtered using a traffic light graphic (red, orange, green) and resulted in eight main indicators and their related action plans. The chapter is very normative and differentiates between three types of knowledge: technical information, local knowledge, and political knowledge as a triad for informing decisions taken to improve children's well-being in communities.

Chapters Five and Six intersect the construct of CWB with gender and crime, respectively. Sharan B. Merriam in Chapter Five suggests alternative indices than gross domestic product to measure well-being and satisfaction, such as the Gross National Happiness and Social Progress Index which addresses basic human needs, the foundations of well-being, and opportunity. Examples of successful countries in adopting such indices are New Zealand, Switzerland, and Iceland. Another alternative index is that by the Organization for Economic Cooperation and Development (OECD) called the Better Life Index that combines measures of material living conditions such as income, jobs, and housing with measures of quality of life such as health, work-life balance, education, and social connections. The benefits of wellbeing include better health, higher civic engagement, and greater resilience.

David C. Sloane and Hyunsun Choi in Chapter Six highlight the important role of sense of connectivity and social trust, translated into social capital and collective efficacy, in increasing the momentum for the positive dialectic relation between the sense of safety and sense of neighbourliness among residents. Such a positive virtuous cycle in turn propagates a sense of spatial freedom for residents. Sloane and Choi aim to understand safety within a community well-being context by using a working definition of CWB by Sung and Phillips that includes residents' perception of the community, residents' needs fulfillment, observable community conditions, and the social and cultural context of the community. Sloane and Choi acknowledge that community safety involves both subjective perceptions (fear of crime) and an objective reality (crime rates). Their contribution pivots on advocating for fitting safety into the theoretical framework introduced by Sung and Phillips, in Chapter One, under the environmental domain. The intention of Sloane and Choi is to instate the sense of safety as a foundational layer in the pyramid form introduced in the first chapter of the book in such as way that it becomes integral to the four related concepts to CWB. It would consist of the sub-concepts of social capital and collective efficacy.

The book does a good job in relating the different topics covered in the forum by means of the theoretical framework at the opening chapter. Without the opening chapter, it would be difficult for the reader to relate the topics of child well-being, gender, and crime to an overall framework. The book tries to supersede the intersections and inter-relations between the four closely related concepts of physical well-being, happiness, QOL, and community development through a redefinition of community well-being as an umbrella construct that encompasses a systemic relation along the four facets of the pyramid - the human, the social, the economic, and the environmental - as well as a systemic relation along the lower and higher echelons of the pyramid layers (subjective and objective/inductive and deductive relations). The main problem that the book seems to revolve around is that of operationalizing a collective sense of well-being from individual and inter-subjective indices. For example, in the second chapter, the authors differentiate between QOL and CWB on such a basis, which also would justify the use of the umbrella concept of CWB. The closest the authors seem to come to in operationalizing the construct is through an inter-subjective approach rather than an objective one.

Nevertheless, a divide seems to persist between the theoretical part of the book and the empirical part. The first two chapters are discussing ontological and conceptual issues, while the remaining chapters do not make explicit connections to the pyramid framework introduced earlier, except for Chapter Six which highlights the importance of community safety to be included as one of the foundational pillars constitutive of any definition of CWB. The authors of the final chapter thus call for a re-conceptualization of community well-being to include community safety as a prerequisite dimension. The reader is left wondering if the form of a quadrilateral pyramid was the best choice by the authors to comprehensively capture the construct of CWB. Perhaps a generative framework that allows for adding other dimensions, such as political, geographic, cultural, would have been more flexible than the static form of a primal geometric form. Overall, the book is definitely a step towards operationalizing the fluid concept of community well-being by the use of theories such as social capital, human capital, needs theory, and community development. The book should have a wide appeal to researchers, and graduate and undergraduate students in the field of community studies.

\section{CONFLICT OF INTEREST DISCLOSURES}

The author declares that they have no conflicts of interest.

\section{AUTHOR AFFILIATIONS}

*Department of General Education, Mount Royal University, Calgary, $\mathrm{AB}$, Canada. 